\title{
Biometrical relationships in developing eggs and neonates of Octopus vulgaris in relation to parental diet
}

\author{
Lorenzo Márquez • Daniel Quintana • \\ Antonio Lorenzo $\cdot$ Eduardo Almansa
}

Received: 10 May 2012/Revised: 6 October 2012/Accepted: 11 October 2012/Published online: 25 October 2012

(C) Springer-Verlag Berlin Heidelberg and AWI 2012

\begin{abstract}
Captive Octopus vulgaris adults were fed three mono-diets based on pilchard, crab and squid and allowed to grow until reproduction under controlled temperature. Spawns from each dietary treatment were isolated, and the embryonic development, egg length, width and wet weight, in addition to neonate dry weight, dorsal mantle length and ventral mantle length were monitored. Pilchard-diet spawns developed faster in terms of thermal time. Initial egg wet weight was higher for squid and crab diets. Irrespective of the parental diet, eggs passed through a swelling process so that egg width and wet weight increased in a nonlinear way, whereas egg length was left nearly unaffected. Egg length and initial wet weight showed a high correlation with neonate dry weight. Egg length, even at advanced incubation, can be used as a good proxy for neonate dry weight, this fact having potential implications for the ecological and aquaculture research on O. vulgaris.
\end{abstract}

Communicated by H.-D. Franke.

L. Márquez ( $\bowtie)$

Dpto. de Biología Aplicada, Escuela Superior de Ingeniería, Universidad de Almería, Ctra. Sacramento s/n. La Cañada de San Urbano, 04120 Almería, Spain

e-mail: marquez.lorenzo728@gmail.com

D. Quintana

UR1067 Nutrition, Métabolisme, Aquaculture, INRA, 64310 Saint-Pée-sur-Nivelle, France

\section{A. Lorenzo}

Dpto. de Biología Animal, Fac. de Biología,

Universidad de La Laguna, 38271 Tenerife, Spain

E. Almansa

Centro Oceanográfico de Canarias (IEO),

Crta. de San Andrés no 45, CP. 38180 S/C de Tenerife, Spain
Keywords Octopus vulgaris $\cdot$ Egg biometry $\cdot$ Paralarvae biometry · Parental diet - Embryonic development .

Thermal time scales

\section{Introduction}

The variation of egg size and its correlates is a recurrent research subject in ecology (Hutchinson 1978; Begon et al. 1996; Koops et al. 2003). Nevertheless, limited environmental or experimental research on egg size has been conducted in cephalopod species. Factors like female size in intra-species comparisons (Laptikhovsky and Nigmatullin 2005), or habitat and mode of life of the hatchlings in inter-species comparisons (Boletzky 1977), partially account for egg size variation, and a West-East differentiation in egg size has been found for some species of the superfamily Argonautoidea in the Mediterranean Sea (Laptikhovsky and Salman 2003). Because yolk synthesis depends upon female metabolism, maternal diet can be thought of as an important factor on cephalopod egg size, but the experimental evidence is controversial. The investigation of the effect of food ration on the reproductive output of the small, multiple-spawning sepioid Idiosepius pygmaeus, resulted in significant differences in the number of egg batches per female, total number of eggs laid and dry weight of the total number of eggs laid, but no difference in the average dry weight per egg was found (Lewis and Choat 1993); on the contrary, food ration had a significant effect on Euprymna tasmanica egg volume: those ones from high-ration females being larger (Steer et al. 2004). Parental diet quality during first feeding has been proven to be an important factor determining egg and hatchling wet weight in Sepia officinalis, since breeders fed a mysid-based diet during the first 20 days of the life cycle 
produced larger eggs and hatchlings than those ones fed an Artemia-based diet (Domingues et al. 2001). No comparable data are available on Octopus vulgaris, although being a second model species in cephalopod research. The natural diet of adult $O$. vulgaris mainly comprises decapod crustaceans, molluscs (including cannibalism) and fish, but a seasonal or habitat-related dietary pattern arises from the analysis of stomach contents or near-den food discards (Caveriviére 2002). Given these data, it is pertinent to investigate the variation in $O$. vulgaris egg size and whether maternal diet could partially account for the potential variability. This is one of the aims of the present work.

To investigate egg size in cephalopods, the egg-swelling process taking place during the embryonic development should be taken into account. Such a fact is a general feature of cephalopod embryonic development, since it has been proven for a wide range of species: Sepia subaculeata (Choe, 1966), Idiosepius pygmaeus (Natsukari 1970), Sepiella japonica (Gomi et al. 1986), Loligo vulgaris and Loligo forbesi (Boletzky 1987), Sepia officinalis (Bouchaud and Daguzan 1990), Todarodes pacificus (Bower and Sakurai 1996), Illex argentinus (Sakai et al. 1998), Illex coindetii (Villanueva et al. 2011), Octopus mimus (Warnke 1999), Sepia apama (Cronin and Seymour 2000), Loligo sanpaulensis (Barón, 2003), Sepiotheutis australis (Steer et al., 2003), O. vulgaris (Silva and Vidal 2006), Dosidicus gigas (Staaf et al. 2008) and Robsonella fontaniana (Uriarte et al., 2009). A $68 \%$ increase in wet weight was attained by $O$. vulgaris eggs incubated for 21 days at $24^{\circ} \mathrm{C}$, whilst dry weight remained nearly constant (Silva and Vidal 2006). The swelling process usually begins at mid or late embryonic development; the earlier stages are nearly constant in size and/or weight, or they go through a shrinking process in the case of some sepiid species (Choe 1966; Gomi et al. 1986; Bouchaud and Daguzan 1990).

Swelling and shrinking processes can potentially erode size variation patterns related to environmental or experimental factors. In the present article, this topic has been investigated by means of two physiological time scales: the Naef scale of embryonic development (Naef 1928) which is a discrete scale based on the embryo morpho-anatomy, and a continuous 'thermal time' scale. In ectotherms, passing of physical time is not meaningful by itself without a reference to temperature; this is why a combination of physical time and temperature, called 'thermal time', is ubiquitously used in the field of entomology to describe processes such as egg development. Thermal time can be briefly defined as the product of time and temperature above a certain temperature threshold (th) for which the process rate is null (Jarosik et al. 2004). In addition, thermal time scales are based on the existence of linear relationships between process rates and thermal time; under these conditions, it is possible to remove much of the variation in the process rate due to temperature. In relation to embryonic development, some discussion exists about the underlying nature of the function linking the process rate and thermal time, but it is generally accepted that such a function fits quite well into a linear pattern (Charnov and Gillooly 2003).

In the particular case of cephalopod eggs, thermal time scales are scarcely used (but see Uriarte et al. 2012), although morpho-anatomical scales of the embryo are excellent physiological-temporal references for biochemical processes taking place during the embryonic development. Nevertheless, there is no clear reason for the underuse of the concept of thermal time in cephalopod research, and in addition, research questions concerned with the rate or duration of the embryonic development will be much more easily treated and understood in terms of thermal time, specially when faced with a scenario of global warming. Since the present investigation is concerned with questions about the course of the incubation process or of the egg biometry, a thermal time approach was applied to obtain appropriate descriptions on a temperature-independent basis, provided that the variation of initial egg size is marginally important in this case (Steer et al. 2002).

The relationship between egg size and newly hatched individual size, within or among species, has been extensively researched in fishes, leading to a general positive correlation (Coleman and Galvani 1998; Chambers and Leggett 1996). Environmental studies have shown that patterns of fish larval size can follow seasonal or latitudinal clines of temperature (Llanos-Rivera and Castro 2004; Hinckley 1990). Such variability is partially explained by maternal genetic background homozygosity in Oreochromis niloticus (Müller-Belecke 2005). In the case of cephalopods, it is well known that egg size is positively correlated to newly hatched individual size when comparing different species and that size at hatching is strongly associated with habitat and mode of life of young specimens (Boletzky 1977). Nevertheless, since inter-species comparisons are expected to be dependent on variation in adaptations gained through evolutionary time (Rocha et al. 2001), the existence of a general inter-species correlation between egg and paralarval biometry does not necessarily imply intra-species correlations. As in the fish case, parental diet seems to positively influence egg size and individual size at hatching in $S$. officinalis (Domingues et al. 2001; 2003); this latter variable determines subsequent growth rate in Octopus pallidus hatchlings, but in complex interaction with temperature (Leporati et al. 2007). At last, variation in size at hatching has already been suggested as an important factor partially explaining the high size-at-age variability that characterises most 
cephalopods populations and this effect has been modelled (Pecl et al. 2004). Therefore, the last aim of the present investigation is to determine to what extent different egg biometrical variables predict paralarval biometrical variables in the case of $O$. vulgaris fed different parental diets.

\section{Materials and methods}

Parental stock rearing conditions and experimental design

Fifty-four $O$. vulgaris adults, 29 females and 25 males, were collected during November 2003 along the Huelva coastal region (SW Spain) by an artisanal fishing boat using cephalopod pots. Female and male mean wet weights were $789.0 \pm 73.9$ and $801.9 \pm 70.5 \mathrm{~g}$, respectively. Adults were stocked in 6 breeding tanks $(\sim 5,000 \mathrm{~L})$ at the Centro IFAPA 'Agua del Pino' aquaculture facilities. The stocking density was 9 individuals per tank. Sex ratio 29:25 was not statistically different from 1:1 (Chi square test with Yates correction for continuity: $\chi^{2}=0.296, d f=1$, $p>0.05)$. Broodstock rearing and spawning period lasted from November to July. A flow-through system of seawater filtered to $25 \mu \mathrm{m}$ was established with a turnover rate of $144 \%$ of the tank volume per day. Animal disturbance were kept at a minimum, except for feeding and cleaning procedures. Water temperature of the breeding tanks rose from 15 to $20{ }^{\circ} \mathrm{C}$, salinity ranged from 35 to 39 psu, and photoperiod was natural and attenuated. Approximately, one pot per animal was placed into each breeding tank, and survival was high $(\sim 80 \%)$. Dens were frequently checked, and spawning females were isolated and maintained at $20 \pm 2{ }^{\circ} \mathrm{C}$ until egg hatching, whereas salinity and photoperiod were as in the breeding tanks.

Three dietary treatments were established: crabs (Carcinus maenas), squids (Illex sp.) and pilchards (Sardina pilchardus); each diet was assigned to 2 breeding tanks. Animals were fed once in the morning. The initial ration was approximately set at $5 \%$ of the biomass per tank (360 g of food per tank). Daily ration was periodically adjusted to the minimum quantity in excess. In this way, food ration was determined by animal demands on food, and at the same time, water quality was well preserved. Tanks fed the same diet received the same daily ration.

Sampling and biometrical procedures

Females were allowed to provide parental care to the eggs. Each spawn was sampled at days 10, 20 and 30 after isolation. Each sampling day, several egg strings were extracted from the spawning pot; afterwards, three strings were randomly chosen for biometrical measurements.
Ten eggs were chosen from string 1 to measure length $(L)$ and width $(l)$ under X40 magnification (Nickon Labophot microscope). Width was taken at the equatorial plane, and length was measured excluding the peduncle. Three string pieces (one from each string) were dissected and weighed, the string weight was subtracted and the obtained figure divided by the number of attached eggs to obtain a mean wet weight (WW). Surface area $(S)$ and volume $(V)$ of eggs were estimated assuming that eggs are prolate ellipsoids, thus:

$$
\begin{aligned}
S & =2 \pi\left(\frac{l}{2}\right)^{2}\left(1+\frac{\operatorname{Larcsin}(e)}{l}\right) ; \quad e=\sqrt{1-\frac{l^{2}}{L^{2}}} \\
V & =\frac{4}{3} \pi \frac{L}{2}\left(\frac{l}{2}\right)^{2}=\frac{\pi L l^{2}}{6} \\
\frac{S}{V} & =3\left(\frac{1}{L}+\frac{1}{l} \frac{\arcsin (e)}{e}\right)
\end{aligned}
$$

where $L$ is the length, $l$ the width of the egg and $e$ is the eccentricity. Eggs were inspected under microscope $(40 \times$ and $100 \times$ magnifications), photographed, and developmental stage was assigned according to Naef scale (Naef 1928). When a precise decision was not possible, a minimum and a maximum stage was deduced and the egg sample was assigned the interval mid-point. A few strings showed eggs in apparently different stages and were discarded.

One-day productions of neonatal paralarvae were gently extracted from isolation tanks by siphoning into a cylindrical methacrylate flask. Ten paralarvae per spawn were randomly chosen for biometrical measurements. Each animal was placed onto a concave slide and exposed to seawater containing a couple of drops of a $\mathrm{MgCl}_{2} 0.41 \mathrm{M}$ solution until swimming movements stopped, and soon after that, ventral mantle length (VML) and dorsal mantle length (DML) were measured under X40 magnification (Nickon Labophot microscope). Dry weight (DW) was measured to avoid variability in water contained into the paleal cavity. To measure palarlarval mean dry weight: (1) Whatman GF/C fibre-glass filters $(4,7 \mathrm{~cm} \varnothing)$ were washed, drained, burnt at $450{ }^{\circ} \mathrm{C}$ for $4 \mathrm{~h}$, dried in silica gel for $45 \mathrm{~min}$ and weighed to the nearest $0.1 \mathrm{mg}$, (2) batches of 40 paralarvae were placed on Whatman filters, washed with ammonium formate $0.5 \mathrm{M}$ and dried at $100{ }^{\circ} \mathrm{C}$ for $24 \mathrm{~h}$, (3) batch weight was obtained as the weight of each filter plus dried paralarvae minus the weight of the filter and (4) a mean dry weight per paralarvae was obtained by dividing by 40 .

To summarise, samples from 4 spawns per diet were analysed. Whenever possible, a maximum of 3 egg samples, comprising several strings, were analysed from each spawn (days 10, 20 and 30); from each egg sample, 10 eggs were measured ( $L$ and $l$ ) and 3 determinations of the 
average egg wet weight were obtained. Nine spawns produced paralarvae: 3 from crab, 2 from squid and 4 from pilchard diet; for each spawn, 10 paralarvae were measured (DML and VML) and 40 ones were used to determine the average dry weight.

Standard thermal time scale for embryonic development of $O$. vulgaris based on Boletzky's data

Thermal time is measured in 'days-degrees' $\left(\mathrm{d}-{ }^{\circ} \mathrm{C}\right)$ and calculated from the following expression (Gilbert et al. 1976):

$\mathrm{tt}=\sum_{t=0}^{d}\left(T_{t}-\mathrm{Th}\right)$

where $t$ is time is days, $t \mathrm{t}$ is the thermal time in day ${ }^{\circ} \mathrm{C}, T_{t}$ is the temperature at day $\mathrm{t}$ in ${ }^{\circ} \mathrm{C}$ and $\mathrm{Th}$ is a threshold temperature in ${ }^{\circ} \mathrm{C}$. In the present work, incubation temperature was nearly constant for one-day periods; thus, Eq. 1 was suitable to estimate thermal times. Whole-spawn thermal times were calculated from the: (1) daily temperatures inside incubation tanks and (2) dates for the onset of the spawning behaviour.

The threshold temperature, Th, was calculated in the present work from previous data published by Boletzky (1974). This author provided with data on the relationship between egg embryonic stage and time in $O$. vulgaris under several constant temperatures $\left(13,15,20\right.$ and $\left.25^{\circ} \mathrm{C}\right)$. Based on these raw data, we applied Eq. 1 to each even Naef stage (II, IV, VI, ..., XX) and so obtained a threshold temperature for each of these Naef stages (Fig. 1a); the average threshold temperature $\left(\mathrm{Th}=10.2^{\circ} \mathrm{C}\right)$ was used to build a general thermal time scale for the embryonic development in O. vulgaris; finally, time $X$-axes in Boletzky's curves (1974) were transformed into a single thermal time $X$-axis, and a cubic polynomial model was fitted to the data (Fig. 1b, Boletzky's curve hereafter), yielding the equation $\left(R^{2}=0.987\right)$ :

$\mathrm{Ns}=-0.22+0.05 \cdot \mathrm{tt}+2 \times 10^{-4} \cdot \mathrm{tt}^{2}-5 \times 10^{-7} \cdot \mathrm{tt}^{3}$

\section{Statistical analyses}

A principal component analysis (PCA) was performed on a subpopulation of $26 \mathrm{egg}$ samples (from 3 crab-diet, 3 squid-diet and 4 sardine-diet spawns) for which all the biometrical $(L, l, W)$ and physiological time variables $(\mathrm{Ns}, \mathrm{tt})$ were recorded. Factors with eigenvalues higher than 0.5 were extracted using the correlation matrix, and a Varimax rotation was performed to clarify the interpretation of the factors. The ratio of cases/variables was 5.2, which is the minimum acceptable ratio for PCA (Hair et al. 1999).
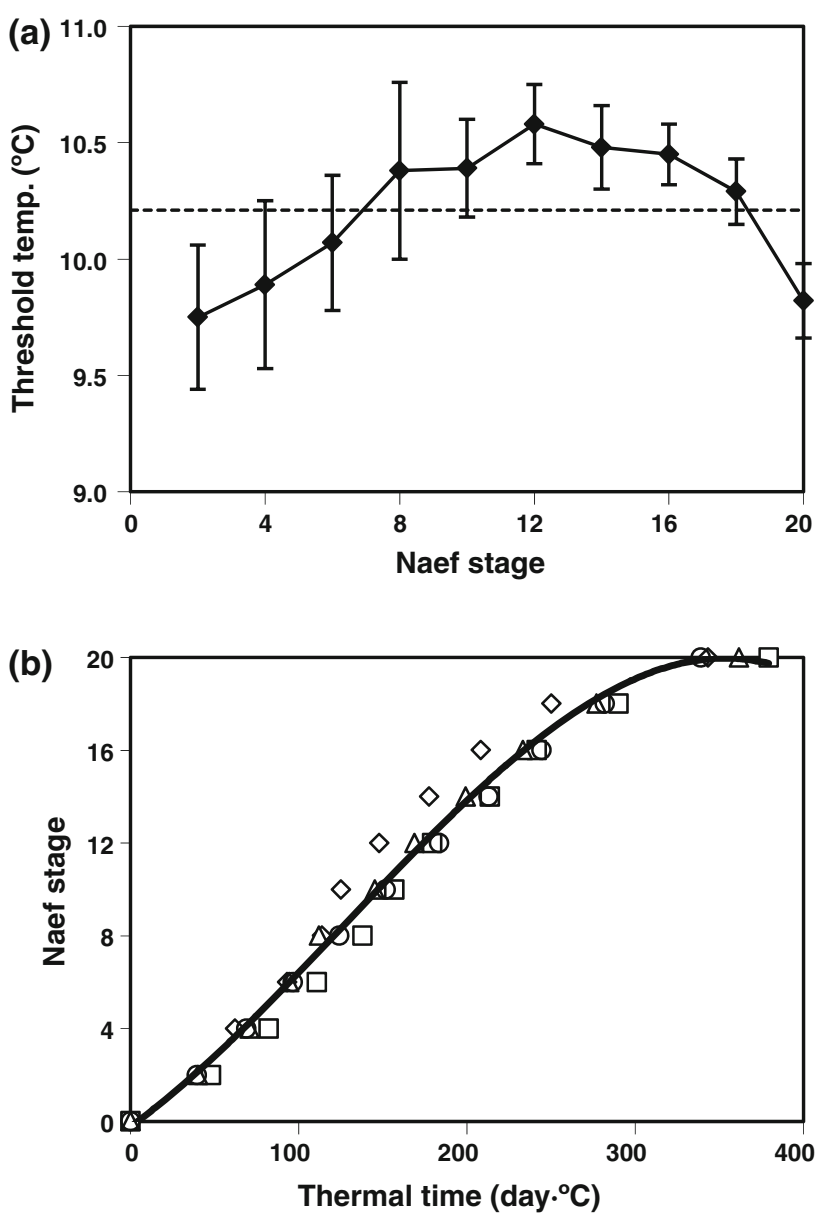

Fig. 1 Thermal time scale for O. vulgaris development based on Boletzky (1974). a Threshold temperatures for different Naef stages; bars SE obtained by nonlinear regression; dashed line represent the average threshold temperature. b Relationship between Naef stage and egg thermal time at different incubation temperatures; diamonds $25{ }^{\circ} \mathrm{C}$; squares $20^{\circ} \mathrm{C}$; triangles $15{ }^{\circ} \mathrm{C}$; circles $13{ }^{\circ} \mathrm{C}$; the black line represents a cubic polynomial fitting

Because biometrical variables were subjected to the joint effects of categorical (diet) and continuous (Naef stage or thermal time) factors, Ancova was the chosen statistical test for data analysis. In addition, as embryonic evolution of egg biometrical variables was markedly curvilinear, a nonparametric version of the test (Ancova on ranks) was performed (Quinn and Keough 2002). Ancova's were performed in a two-step way: firstly, lack of factor interaction was tested (equivalent to slope homogeneity in parametric Ancova), and secondly, if no significant interaction was found, factor effects were tested.

Local weighted regression (Lowess) was used in the present study to visually determine the evolution of egg biometrical variables throughout embryonic development. Lowess is a nonparametric regression method useful for smoothing noisy plots; no regression equation is provided, but a robust trend line can be obtained for the relationship between two variables (Quinn and Keough 2002). 


\section{Results}

Spawn incubation process in relation to thermal time scales

Following the principle that thermal time (tt) can be used to describe development of eggs, we have also applied this rule to describe the physiological course of a whole spawn. When this is done for all egg samples within a given dietary treatment, and Naef stage is plotted versus sample tt, the following results are apparent (Fig. 2): (1) some egg samples stand well below the Boletzky's curve, (2) the variability in embryonic stage seems to increase from 0 to approximately $200 \mathrm{~d}-{ }^{\circ} \mathrm{C}$, and then, it decreases until approximately $385 \mathrm{~d}-{ }^{\circ} \mathrm{C}$, when hatching takes place, (3) very early developmental stages $\left(\leq\right.$ Naef II) were found until $200 \mathrm{~d}-{ }^{\circ} \mathrm{C}$ for crab-diet and squid-diet spawns but not for sardine-diet spawns, (4) when the relationship between egg developmental stage and spawn thermal time is showed by a Lowess regression the obtained line is below Boletzky's curve, particularly for crab and squid dietary treatments.

Scaling egg samples by principal component analysis (PCA)

Two factors with eigenvalues above 0.5 were extracted by the PCA; the cumulative variance explained was higher than $90 \%$. After a varimax rotation (Fig. 3b), Factor 1 was more related to any of the physiological time variables

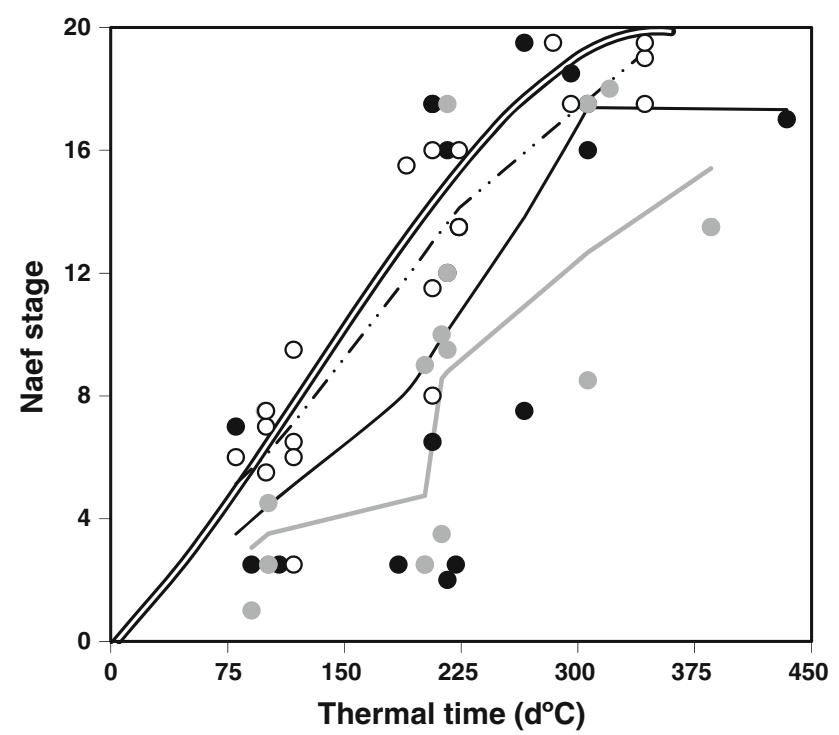

Fig. 2 Egg embryonic stage versus whole-spawn thermal time. Double black line is Boletzky's curve. Black circles crab diet; grey circles squid diet; open circles pilchard diet. Black line Lowess regression for crab diet; grey line Lowess regression for squid diet; dashed line Lowess regression for pilchard diet. Window for Lowess regression equals 0.8
$(\mathrm{Ns}, \mathrm{tt})$ and Factor 2 to egg length $(L)$; the loadings of egg width and wet weight $(l, \mathrm{ww})$ were intermediate in both factors. A plot of factor scores for each egg sample (Fig. 3a) separates the majority of sardine samples from the rest and establishes a clear separation between samples bellow and above Naef stage 10 .

As expected, the variables $\mathrm{Ns}$ and $\mathrm{tt}$ are redundant according to PCA, so that one or the other can be used to represent the physiological state of the eggs.

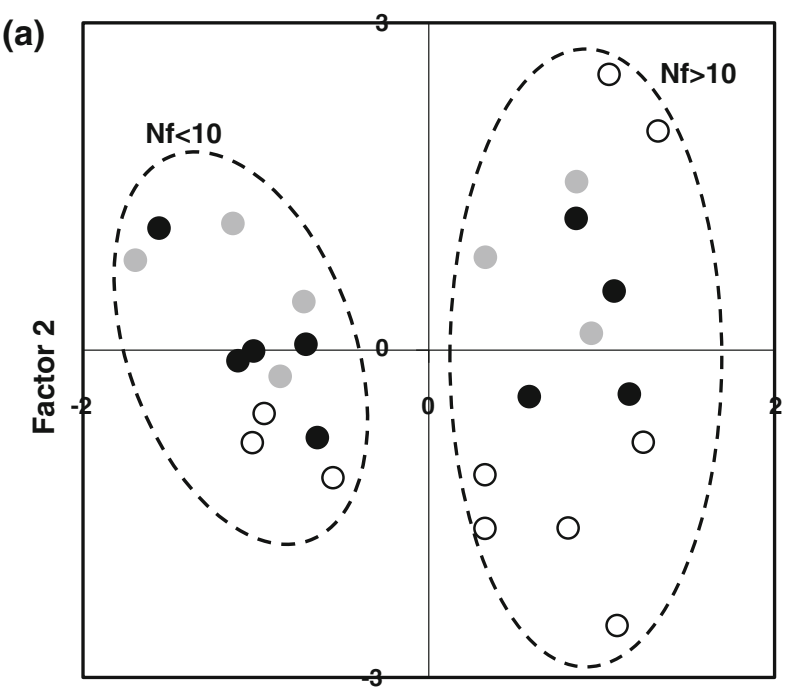

Factor 1

(b)

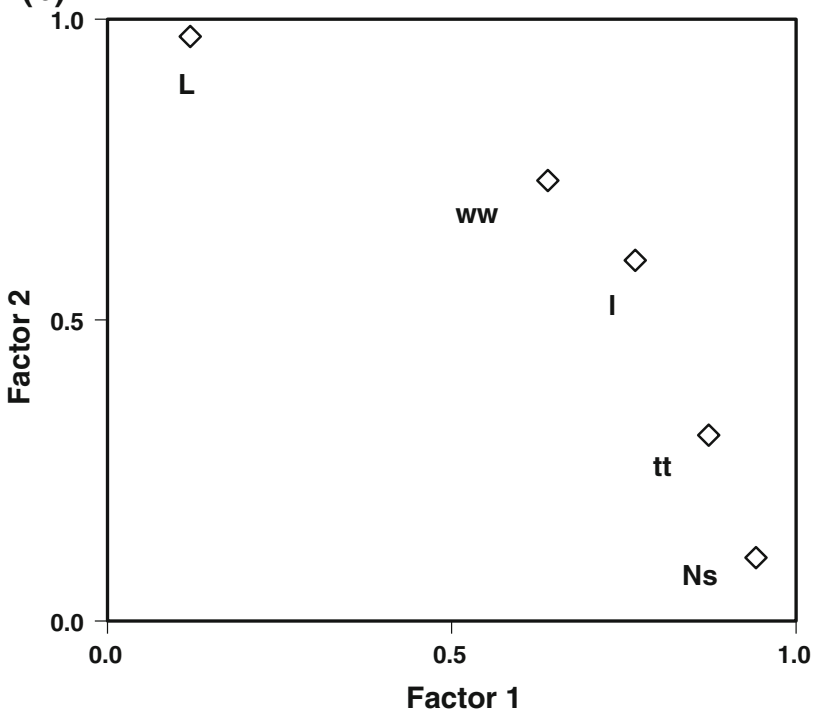

Fig. 3 Ordination of spawns by a principal component analysis on egg samples. a Case factor-coordinate plot; black circles crab diet; grey circles squid diet; open circles pilchard diet. The two ellipses separate samples according to their Naef stage. b Variable factorcoordinate plot; $t t$ thermal time, $N s$ Naef stage, $L$ mean egg length, $l$ mean egg width, $W W$ mean egg wet weight 
The effects of maternal diet and development stage on egg biometry

Since PCA showed that Ns and tt were redundant variables, the first one was selected to analyse the effects of physiological time. In a nonparametric two-way ANOVA on egg biometry by maternal diet and Naef stage, the only factor that statistically affected egg length $(L)$ was maternal diet (Table 1). The eggs from sardine diet were shorter than those ones from squid or crab diets. In the case of egg width $(l)$, the only significant factor was the embryonic stage. Egg width increased through embryonic development and S/V decreased (Fig. 4). At last, egg mean wet weight (WW) was influenced by both embryonic stage and maternal diet, increasing throughout embryonic development for any of the tested diets.

The evolution of $1, \mathrm{~S} / \mathrm{V}$ and WW throughout embryonic development was not linear, but it can be described as two consecutive phases, as shown by Lowess regression (Fig. 4). The first phase lasted from Naef stage 1 to some point between Naef stages 10 and 15; within this first phase, a slow swelling process was apparent. Afterwards, a slope change was apparent and a second linear phase characterised by an accelerated egg swelling was recorded. The swelling process proceeded so that the mainly affected variable was egg width $(l)$, leaving egg length $(L)$ nearly unchanged and eggs becoming more rounded in shape. As a consequence, the $S / V$ ratio decreased and, at the same time, egg mean wet weight (WW) increased. Lowess regression suggested that in the second phase of the swelling process, the WW versus embryonic stage regression line had a steeper slope in the sardine diet when compared to slopes of squid and crab diets, but no statistical interaction was found. Therefore, the whole eggswelling pattern described above appeared to be quite independent of the maternal diet: parallel, two-phased regression lines can be suggested for different maternal diets, being the line for the sardine diet the lower located one and existing no statistical differences between the squid-diet and crab-diet lines (data shown in Fig. 4; analysis shown in Table 1).

A plot of WW versus tt (instead of Ns as the physiological time variable) is presented in Fig. 4e. Only egg samples whose Naef stage were not further than \pm 3 stages from those predicted by Boletzky's curve were used in Fig. $4 \mathrm{e}$, since their thermal times can be estimated by taking the beginning of the spawning behaviour as the actual time origin. Twenty-seven egg samples met this imposed requirement, and a nonparametric two-way ANOVA on egg WW by parental diet and thermal time showed no significant effect of parental diet $(d f=2$, $\chi^{2}=2.51, p=0.285$ ). As mentioned above, if Ns is used as the physiological time variable, the same ANOVA
Table 1 Nonparametric Ancova's on biometrical variables of $O$. vulgaris eggs by parental diet and embryonic stage

\begin{tabular}{|c|c|c|c|c|c|}
\hline \multirow{2}{*}{$\begin{array}{l}\text { Egg } \\
\text { variable }\end{array}$} & \multirow[t]{2}{*}{$N$} & & \multicolumn{3}{|l|}{ Factor } \\
\hline & & & $\begin{array}{l}\text { Parental diet } \\
d f=2\end{array}$ & $\begin{array}{l}\text { Naef stage } \\
d f=1\end{array}$ & $\begin{array}{l}\text { Interaction } \\
d f=2\end{array}$ \\
\hline \multirow[t]{2}{*}{$L$} & \multirow[t]{2}{*}{30} & $\chi^{2}$ & 7.21 & 1.84 & 1.12 \\
\hline & & $p$ & 0.007 & 0.175 & 0.571 \\
\hline \multirow[t]{2}{*}{$l$} & \multirow[t]{2}{*}{30} & $\chi^{2}$ & 2.67 & 18.23 & 0.820 \\
\hline & & $p$ & 0.263 & $<0.001$ & 0.664 \\
\hline \multirow[t]{2}{*}{ WW } & \multirow[t]{2}{*}{49} & $\chi^{2}$ & 8.51 & 25.18 & 0.810 \\
\hline & & $p$ & 0.014 & $<0.001$ & 0.667 \\
\hline
\end{tabular}

Bold values indicate significant correlations

disclosed a significant effect of parental diet, probably because the sample with suitably assigned physiological times nearly doubled (49 egg strings). Interestingly, if the relationship between WW and $\mathrm{tt}$ is represented by a Lowess regression line for pooled data (irrespective of parental diet), the egg WW reported by Silva and Vidal (2006) about Brazilian O. vulgaris development is precisely predicted.

Biometry of newly hatched paralarvae and correlations with egg biometrical variables

Correlation between egg and paralarval biometry was tested using different egg-paralarva pairs of biometrical variables; $L, l$ and WW at day 10 (approximately $100 \mathrm{~d}-{ }^{\circ} \mathrm{C}$ ) were used as egg variables, and DML, VML and DW was used as paralarval variables. The main results (Table 2, Fig. 5) were as follows: (1) paralarval dry weight showed a high correlation to egg $L$ and WW and (2) egg breadth (l) provided very poor correlations when compared to paralarval DW. Additionally, a one-way Anova on paralarval mean DW by parental diet showed a significant effect $(p=0.003)$, paralarvae from pilchard diet being smaller than those from crab diet or squid diet $(0.23,0.29$ and $0.31 \mathrm{mg}$, respectively).

\section{Discussion}

Egg biometry as a proxy for paralarval biometry in O. vulgaris: implications for basic and applied research

Offspring biometrical variables are much more easily measured than biochemical ones. The correlations between egg length, width or weight and the mean paralarval dry weight (DW) are actually used in some of the aforementioned articles (Table 3). The suitability of biometric variables to compare different spawns will depend on their 
Fig. 4 Variation of biometrical egg variables throughout embryonic development of O. vulgaris. Lines stand for Lowess regressions with a window of 0.6: for pooled data when the effect of diet was not significant, otherwise for each diet; black circles and lines crab diet; grey circles and lines squid diet; open circles and dashed lines sardine diet. In plot $\mathbf{e}$, samples with eggs within \pm 3 Naef stages from Boletzky's curve were used; large crosses represent data from Silva and Vidal (2006) (a)

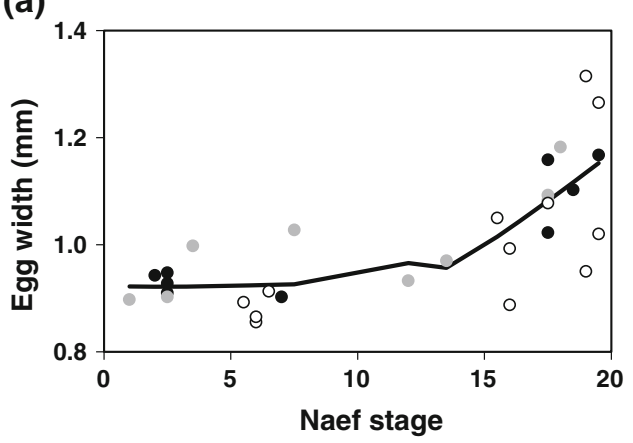

(c)

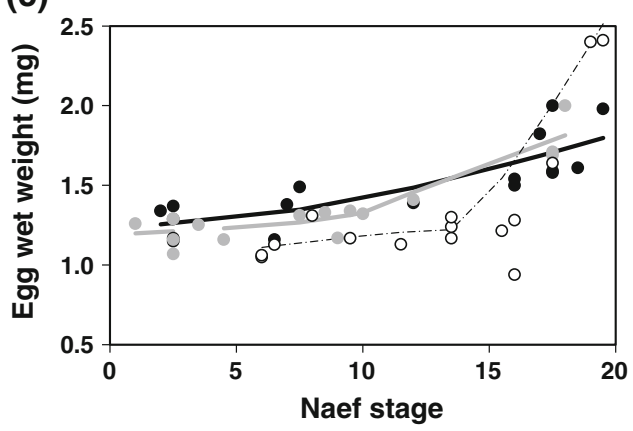

(e)

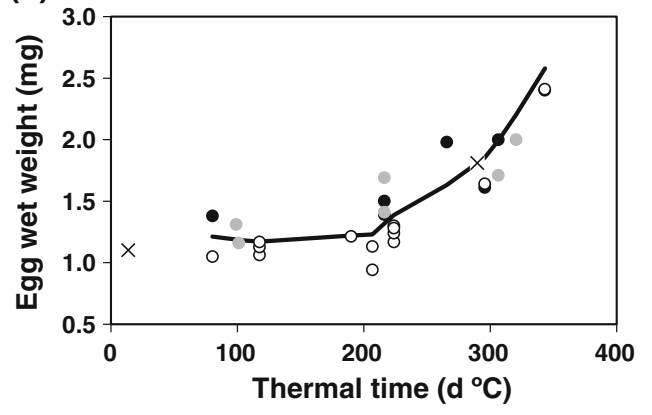

(b)

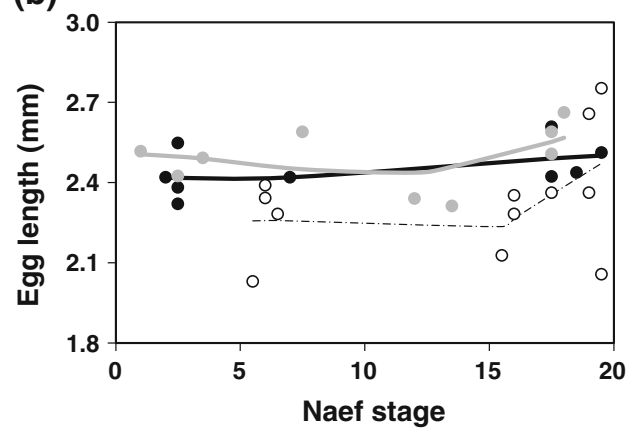

(d)

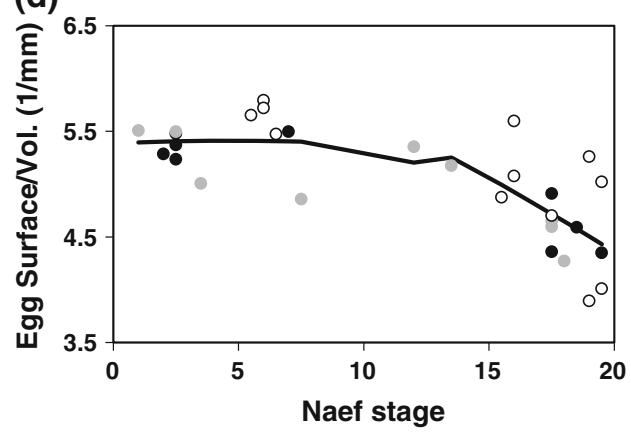

Table 2 Spearman's correlations between mean values per spawn of biometrical variables measured in eggs at day 10 of incubation and in paralarvae at hatching

\begin{tabular}{lllllr}
\hline \multirow{2}{*}{$\begin{array}{l}\text { Egg } \\
\text { variables }\end{array}$} & $N$ & Spearman correlation & \multicolumn{3}{c}{ Paralarval variables } \\
\cline { 4 - 6 } & & & DML & VML & DW \\
\hline$L$ & 9 & Rho & 0.233 & 0.100 & $\mathbf{0 . 9 0 0}$ \\
& & $p$ & 0.546 & 0.798 & $\mathbf{0 . 0 0 1}$ \\
$l$ & 9 & Rho & 0.333 & 0.183 & 0.567 \\
& & $p$ & 0.381 & 0.637 & 0.112 \\
WW & 9 & Rho & 0.483 & 0.267 & $\mathbf{0 . 9 3 3}$ \\
& & $p$ & 0.187 & 0.488 & $<\mathbf{0 . 0 0 1}$ \\
\hline
\end{tabular}

Bold values indicate significant correlations

variances and stabilities throughout the embryonic development. Out of the five variables analysed in Fig. 4, two could distinguish among dietary treatments, that is, egg length and wet weight, and only egg length showed an extended period of constancy, from Naef stage 1 to approximately Naef stage 15 . It is interesting to note that, in the case of the octopodid $R$. fontaniana, a period of egg length constancy was apparent from day 0 to approximately 35 days when incubation proceeded at $12{ }^{\circ} \mathrm{C}$, that is, until certain Naef stage between $\mathrm{XI}$ and $\mathrm{XV}$ (Uriarte et al. 2009), which is compatible with our data on $O$. vulgaris. Thus, it may be suggested that the more suitable biometric variable to compare egg sizes among spawns of $O$. vulgaris is egg length and, possibly, the same could be true for $R$. fontaniana.

The biometrical results presented in this work make it possible to predict the paralarval size of $O$. vulgaris at hatching from egg size (Fig. 5). The correlation found between egg capsule length and paralarval dry weight is especially interesting, since it provides a way to predict paralarval weight which is independent of the embryonic stage of the egg, except for the last stages. This would be useful in field research, allowing an estimation of neonate dry weight from egg length in the wild, where it is difficult 


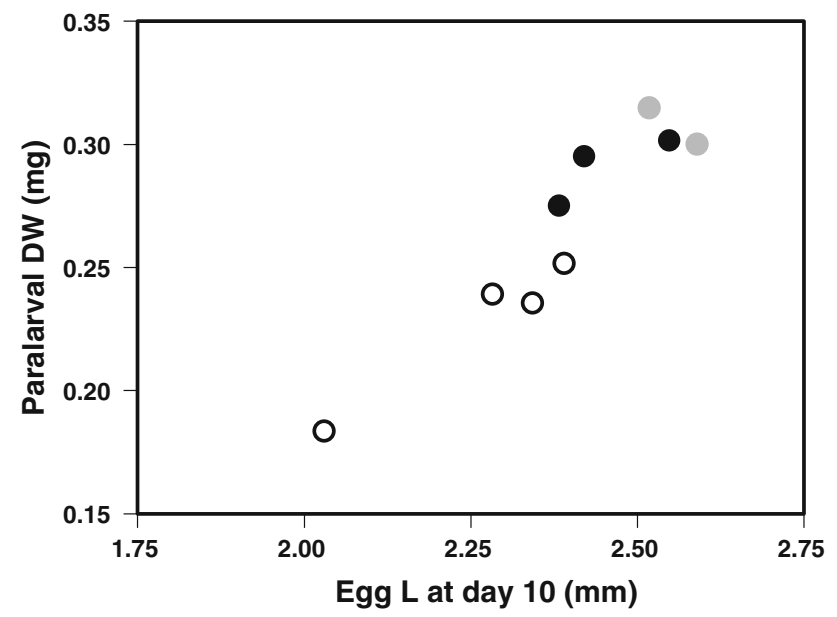

Fig. 5 Relationship between egg length $(L)$ at day 10 and newly hatched paralarva dry weight $(D W)$; black circles crab diet; grey circles squid diet; open circles sardine diet

to find planktonic stages. If a coastal spawning zone is identified in the wild, it could be possible to periodically collect egg samples for one or several spawning seasons. Procedures used by artisanal fishermen to capture common octopus, consisting in artificial dens tied to lines and spread over the sea bottom, could perhaps help in the in situ collection and sampling of spawning females. Egg length, together with the regime of local temperatures during the spawning season, would determine paralarval size at hatching according to the present results.

Another possible application of this biometrical correlation is in aquaculture research; $O$. vulgaris is presently under active investigation in order to achieve an efficient paralarval culture protocol; thus, predicting paralarval weight from egg features could be useful to the early assessment of spawning quality. Nevertheless, some restrictions must be kept in mind when applying the correlations found, mainly because resting metabolism increases with temperature (Clarke and Fraser 2004), so a larger portion of the yolk will be spent in embryonic catabolism at higher temperatures, reducing embryonic growth (Bouchaud and Daguzan 1990). In aquaculture facilities, temperature is usually under control, but the 'thermic history' of natural spawnings, jointly with egg size, will be important to determine paralarval size at hatching; this restriction will be more stringent when comparing spawnings from a wide spatial or temporal range.

Spawn incubation process in relation to physiological time scales

It is interesting to note that, when the incubation progress is represented by a Lowess regression of egg stage versus spawn thermal time, the obtained line is always below the Boletzky's curve (Fig. 2), the gap being larger for crab and squid dietary treatments. On the one hand, the discrepancy between Boletzky's curve and Lowess lines could be explained by the asynchrony in egg-laying (Wells, 1978), so that the egg mass incubation can be viewed as a dynamic process following the principle first-in, first-out. Provided that $O$. vulgaris egg-laying behaviour for a single spawn lasts over 7 days (Wells 1978), a mixture of developmental embryonic stages could be found at any moment throughout the incubation period, and only the eggs spawned at the onset of spawning phase will show the maximal developmental stage within any checked sample. On the other hand, when a spawn comprises a reduced number of eggs,

Table 3 Swelling pattern of eggs of different species

\begin{tabular}{llllll}
\hline Species & Family & Var. & Temp. & Pattern & Data source \\
\hline I. pygmaeus & Idiosepiidae & $L, l$ & $18.5-22.6$ & $=+$ & Natsukari (1970) \\
L. forbesi & Loliginidae & $L, l$ & $12-15$ & ++ & Boletzky (1987) \\
L. vulgaris & Loliginidae & $L, l$ & $12-15$ & $=+$ & Boletzky (1987) \\
S. australis & Loliginidae & $V$ & $13 ; 16$ & $=+$ & Steer et al. (2003) \\
I. coindetii & Ommastrephidae & $L$ & 17 & $=+$ & Villanueva et al. (2011) \\
S. subaculeata & Sepiiidae & $L, l$ & $18-28$ & -+ & Choe (1966) \\
S. japonica & Sepiidae & $W$ & $20-22$ & -+ & Gomi et al. (1986) \\
S. officinalis & Sepiidae & $W$ & $12 ; 15 ; 18 ; 21$ & ++ & Bouchaud and Daguzan (1990) \\
$R$. fontaniana & Octopodidae & $W$ & 12 & $=+$ & Uriarte et al. (2009) \\
R. fontaniana & Octopodidae & $L$ & 12 & ++ & Uriarte et al. (2009) \\
O. vulgaris & Octopodidae & $l, V, W$ & $18-22$ & $18-22$ & This article \\
O. vulgaris & Octopodidae & $L$ & This article
\end{tabular}

Biometric variables were made on egg chorions for $O$. vulgaris and I. coindetii and on egg capsules for the rest of species. Temperature in ${ }^{\circ} \mathrm{C}$. Swelling patterns for $O$. vulgaris were assessed on the basis of Ancova's results; for the remaining species, the assessment was based on visual inspection of the published results. Temp. temperature, $L$ egg length, $l$ egg width, $V$ egg volume, $W$ egg wet weight. Swelling pattern: -+ a decreasing phase followed by an increasing phase; $=+$ constancy followed by an increasing phase; ++ a slow increasing phase followed by a fast increasing phase; = constancy all the time 
the thermal time required to complete the spawning process and, subsequently, the thermal time to complete incubation and hatching should be reduced as well.

The effects of maternal diet on spawn incubation progress

At least, three former papers have described the effect of maternal diet and/or ration on cephalopod egg size with heterogeneous results. In the species Euprymna tasmanica, the authors concluded that maternal ration correlated positively with egg size (Steer et al. 2004), whereas no effect of ration on egg size was found in I. pygmaeus (Lewis and Choat 1993) nor in Enteroctopus megalocyathus (Farías et al., 2011); in the last two species, the effect of low rations implied low fecundities instead of smaller eggs. This picture resembles that reported in arthropodfemales within populations, where food-stressed females lay smaller eggs in most of the species, but a number of species produce smaller or equally sized eggs, the reasons for this heterogeneity remaining poorly understood (Fox and Czesak 2000). In the present investigation, the effect of low ingestion rates on egg size is more similar to that found by Steer et al. (2004) in E. tasmanica, despite being difficult to find a simple underlying factor in common to both species: on the one hand, the mode of life of $O$. vulgaris neonates is ecologically more related to those of I. pygmaeus, which are planktonic (Boletzky 2003) and, on the other hand, $O$. vulgaris is phylogenetically closer to E. megalocyathus.

In the present investigation, daily ration was determined by animal demands on food and only the kind of prey was determined by the investigators. As a result, daily ration per tank rose stepwise from $360 \mathrm{~g} \mathrm{tank}^{-1}$ day $^{-1}$ to maximum rations of $1,000 \mathrm{~g} \mathrm{tank}{ }^{-1} \mathrm{day}^{-1}$ and $1,200 \mathrm{~g}$ $\operatorname{tank}^{-1} \mathrm{day}^{-1}$ in the squid and crab diets, respectively, whereas it fell into $200 \mathrm{~g} \mathrm{tank}^{-1}$ day $^{-1}$ after the first week of feeding with sardine and kept that level since then. This is in agreement with the prey preferences published for O. vulgaris in the field (Caveriviére 2002) or in laboratory experiments (Cagneta and Sublimi 2000).

The evolution of egg biometry throughout embryonic development

In $O$. vulgaris, egg chorion width is more prone to vary than egg chorion length during the swelling period, $40 \%$ versus 2-8\% (depending on the diet), respectively (Fig. 4). Similarly, in species with capsulated eggs, the relative variation in egg width is larger than that for egg length: for example, in I. pygmaeus (Natsukari 1970), S. subaculeata (Choe 1966), L. vulgaris (Boletzky 1987) and L. forbesi (Boletzky 1987), egg width increases by a 79, 58, 80 and $87 \%$, respectively, during embryonic development, and the egg length by a $68,45,30$ and $79 \%$, respectively. In any event, in species possessing an egg gelatinous external envelope, the egg-swelling process is related to this envelope (Ikeda et al. 1993; Staaf et al. 2008; Villanueva et al. 2011), this fact suggesting a difference in the underlying mechanism with respect to eggs without this structure, such as those laid by incirrate octopod species.

For six different spawns in the species $O$. mimus, it was found a chorion length increment ranging between 4 and $16 \%$, although 4 out of the 6 studied cases were between 4 and $6 \%$ (Warnke 1999), which is in good agreement with $O$. vulgaris data. This fact suggests a general difference in developmental biometric variation between capsulated and noncapsulated eggs, the former ones suffering a smaller variation in egg length than the latter; nevertheless, more data on noncapsulated egged species are needed.

Cronin and Seymour (2000) found that biometrical variation of eggs of S. apama is important in maintaining a nonlimiting concentration of oxygen in the periviteline fluid throughout the embryonic development. In that respect, the approximately $20 \%$ decrease in surface to volume ratio of eggs in late development here demonstrated, together with the massiveness spawns and the asynchronous development of eggs within a spawn, is expected to be a relevant factor affecting the rate of oxygen uptake in $O$. vulgaris spawns.

Acknowledgments This work was supported by the European Union program: Iniciativa Comunitaria INTERREG III A EspañaPortugal: Proyecto Diveraqua SP5 E36. Daniel Quintana would like to thank the support of the grant provided by the Junta de Andalucía during the period of this research. The authors thank three anonymous referees for helpful criticism.

\section{References}

Barón PJ (2003) Embryonic development of the south American longfin squid Loligo sanpaulensis Brakoniecki, 1984. J Moll Stud 69:221-227

Begon M, Harper JL, Townsend CR (1996) Ecology: individuals, populations and communities. Blackwell Science, Oxford

Boletzky SV (1974) Élevage des cephalopods en aquarium. Vie et Milieu 24:309-340

Boletzky SV (1977) Post-hatching behaviour and mode of life in cephalopods. Symp Zool Soc Lond 38:557-567

Boletzky SV (1987) On egg and capsule dimensions of Loligo forbesi (Mollusca : Cephalopoda): a note. Vie Milieu 37:187-192

Boletzky SV (2003) A lower limit to adult size in coleoid cephalopods: elements of a discussion. Berliner Paläobiol Abh 3:19-28

Bouchaud O, Daguzan J (1990) Étude expérimentale de l'influence de la température sur le déroulement embryonnaire de la seiche Sepia officinalis L. (Cépalopode, Sepioidae). Cah Biol Mar 31: $131-145$

Bower JR, Sakurai Y (1996) Laboratory observations on Todarodes pacificus (Cephalopoda: Ommastrephidae) egg masses. Am Malacol Bull 13:1996 
Cagneta P, Sublimi A (2000) Productive performance of the common octopus (Octopus vulgaris C.) when fed on a monodiet. Cah Options Méditerr 47:331-336

Caveriviére A (2002) Élements du cycle de viedu poulpe Octopus vulgaris des eau sénégalaises. In: Caverivière $A$, Thiam $M$, Jouffre D (eds) Le poulpe Octopus vulgaris. Sénégal et côtes nord-ouest africaines. IRD Éditions, Paris, pp 105-125

Chambers RC, Leggett WC (1996) Maternal influences on variation in egg sizes in temperate marine fishes. Am Zool 36:180-196

Charnov EL, Gillooly JF (2003) Thermal time: body size, food quality and the $10 \mathrm{C}$ rule. Evol Ecol Res 5:43-51

Choe S (1966) On the eggs, rearing, habits of the fry, and growth of some cephalopoda. Bull Mar Sci 16:330-348

Clarke A, Fraser PP (2004) Why does metabolism scale with temperature? Funct Ecol 18:243-251

Coleman RM, Galvani AP (1998) Egg size determines offspring size in neotropical cichlid fishes (Teleostei: Cichlidae). Copeia 1:209213

Cronin ER, Seymour RS (2000) Respiration of the eggs of the giant cuttlefish Sepia apama. Mar Biol 136:863-870

Domingues PM, Sykes A, Andrade JP (2001) The use of Artemia sp. or mysids as food source of the cuttlefish (Sepia officinalis L.); effects on growth and survival throughout the life cycle. Aquacult Int 9:319-331

Domingues P, Sykes A, Sommerfeld A, Andrade JP (2003) Effects of feeding live or frozen prey on growth, survival and the life cycle of the cuttlefish, Sepia officinalis (Linnaeus, 1758). Aquacult Int 11:397-410

Fox CH, Czesak ME (2000) Evolutionary ecology of progeny size in arthropods. Annu Rev Entomol 45:341-369

Gilbert N, Gutiérrez AP, Frazer BD, Jones RE (1976) Ecological relationships. W. H. Freeman and Company Ltd., Reading

Gomi F, Yamamoto M, Nakazawa T (1986) Swelling of egg during development of the cuttlefish, Sepiella japonica. Zool Sci 3:641-645

Hair JF, Anderson RE, Tatham RL, Black WC (1999) Análisis Multivariante $5^{\text {a }}$ ed. Prentice Hall Iberia, Madrid

Hinckley S (1990) Variation of egg size of walleye pollock Theragra chalcogramma with a preliminary examination of the effect of egg size on larval size. Fish Bull 88:471-483

Hutchinson GE (1978) An introduction to population ecology. Yale University Press, New Haven

Ikeda Y, Sakurai Y, Shimazaki K (1993) Fertilizing capacity of squid (Todarodes pacificus) spermatozoa collected from various sperm storage sites, with special reference to the role of gelatinous substance from the oviductal gland in fertilization and embryonic development. Invertebr Reprod Dev 23:39-44

Jarosik V, Kratochvil L, Honëk A, Dixon AFG (2004) A general rule for the dependence of developmental rate on temperature in ectothermic animals. Proc R Soc Lond B (Suppl.) 271:S219S221

Koops MA, Hutchings JA, Adams BK (2003) Environmental predictability and the cost of imperfect information: influences on offspring size variability. Evol Ecol Res 5:29-42

Laptikhovsky VV, Nigmatullin CM (2005) Aspects of female reproductive biology of the orange-back squid, Sthenoteuthis pteropus (Steenstup) (Oegopsida: Ommastrephidae) in the eastern tropical Atlantic. Sci Mar 69:383-390

Laptikhovsky VV, Salman A (2003) On the reproductive strategies of the epipelagic octopods of the superfamily Argonautoidea (Cephalopoda: Octopoda). Mar Biol 142:321-326

Leporati S, Pecl G, Semmens JM (2007) Cephalopod hatchling growth: the effects of initial size and seasonal temperatures. Mar Biol 151:1375-1383
Lewis AR, Choat JH (1993) Spawning mode and reproductive output of the tropical cephalopod Idiosepius pygmaeus. Can J Fish Aquat Sci 50:20-28

Llanos-Rivera A, Castro LR (2004) Latitudinal and seasonal egg-size variation of the anchoveta (Engraulis ringens) off the Chilean coast. Fish Bull 102:207-212

Müller-Belecke A (2005) Relations between egg size, reproductive success and growth performance of progeny in isogenic Oreochromis niloticus lines. Aquaculture 247:127-134

Naef A (1928) Fauna and flora of the Bay of Naples. Cephalopoda embryology. Smithsonian Institution Libraries, Washington

Natsukari Y (1970) Egg-laying behaviour, embryonic development and hatched larva of the pygmy cuttlefish, Idiosepius pygmaeus paradoxus Ortmann. Bull Fac Fish Nagasaki Univ 30:15-29

Pecl GT, Steer MA, Hodgson KE (2004) The role of hatchling size-atage variability of cephalopods: extending the Forsythe hypothesis. Mar Fres Res 55:387-394

Quinn GP, Keough MJ (2002) Experimental design and data analysis for biologist. Cambridge University Press, Cambridge

Rocha F, Guerra A, González AF (2001) A review of reproductive strategies in cephalopods. Biol Rev 76:291-304

Sakai M, Brunetti ME, Ykeda E, Sakurai Y (1998) Embryonic development and hatchlings of Illex argentines derived from artificial fertilization. S Afr J Mar Sci 20:255-265

Silva L, Vidal E (2006) Yolk utilization, water, organic and inorganic content of Octopus vulgaris eggs during embryonic development. In: Abstract book of the Cephalopod International Advisory Council Symposium on Cephalopod Life Cycles, Hobart, Tasmania, 6-10 February 2006. Cephalopod International Advisory Council, Hobart, Tasmania, 102 pp. http://www.abdn.ac.uk/ CIAC/conferences/Program2006.pdf

Staaf DJ, Camarillo-Coop S, Haddock SHD, Nyack AC, Payne J, Salinas-Zavala CA, Seibel BA, Trueblood L, Widmer C, Gilly WF (2008) Natural egg mass deposition by the Humboldt squid (Dosidicus gigas) in the Gulf of California and characteristics of hatchlings and paralarvae. J Mar Biol Assoc UK 88:759-770

Steer MA, Moltschaniwskyj NA, Gowland FC (2002) Temporal variability in embryonic development and mortality in the southern calamary Sepiotheutis australis: a field assessment. Mar Ecol Prog Ser 243:143-150

Steer MA, Moltschaniwskyj NA, Jordan AR (2003) Embryonic development of southern calamary (Sepiotheutis australis) within the constrains of an aggregated egg mass. Mar Fres Res 54:217-226

Steer MA, Moltschaniwskyj NA, Nichols DS, Miller M (2004) The role of temperature and maternal ration in embryo survival: using the dumpling squid Euprymna tasmanica as a model. J Exp Mar Biol Ecol 307:73-89

Uriarte I, Zúñiga O, Olivares A, Espinoza V, Cerna V, Farías A, Rosas C (2009) Morphometric changes and growth rate during embryonic development of Robsonella fontaniana. Vie Milieu 59:315-323

Uriarte I, Espinoza V, Herrera M, Zúñiga O, Olivares A, Carbonell P, Pino S, Farías A, Rosas C (2012) Effect of temperature on embryonic development of Octopus mimus under controlled conditions. J Exp Mar Biol Ecol 416-417:168-175

Villanueva R, Quintana D, Petroni G, Bozzanno A (2011) Factors influencing the embryonic development of and hatchling size of the oceanic squid Illex coindetii following in vitro fertilization. J Exp Mar Biol Ecol 407:54-62

Warnke K (1999) Observations on the embryonic development of Octopus mimus (Mollusca: Cephalopoda) from northern Chile. Veliger 42:211-217

Wells MJ (1978) Octopus. Physiology and behaviour of an advanced invertebrate. Chapman and Hall, London 